\title{
EDUCACIÓN PARA LA SALUD SEXUAL EN EL INSTITUTO: A PROPÓSITO DE UN CASO
}

Sexual health education in High School: a case to study

No Instituto de educação de saúde sexual: um propósito de um caso

\author{
Andrea Aguado Arconada (1) \\ Francisco Javier Pérez Rivera (2) \\ Olga Arias Gundín (3)
}

Elena Andina Díaz (4)

(1) Universidad de León, aaguaa00@estudiantes.unileon.es

(2) Departamento Enfermería y Fisioterapia. Universidad de León.

javier.perez.rivera@unileon.es

(3) Departamento Psicología, Sociología y Filosofía. Universidad de León.

o.arias.gundin@unileon.es

(4) Departamento Enfermería y Fisioterapia. Universidad de León.

elena.andina@unileon.es

\section{Resumen}

Los profesionales sanitarios y de la educación jugamos un importante papel de acompañamiento y guía en la educación sexual de los adolescentes. Además de los modelos tradicionales de enseñanza, hoy se apuesta por metodologías de acción participativas. El objetivo de este trabajo fue fomentar el aprendizaje en dicha materia a alumnos de un instituto, comparando diferentes metodologías educativas. Se impartieron tres talleres educativos (metodología tradicional, role-playing y dináminas), y se evaluó la eficacia de cada uno de ellos, valiéndonos de un cuestionario pretest y postest. Se constató la importancia de la educación sexual en adolescentes en el ámbito académico, y se demostraron algunas fortalezas y debilidades de las metodologías tradiciones y participativas en dicho proceso.

Palabras clave: Educación para la salud; Sexualidad; Adolescentes 


\begin{abstract}
Health and education professionals play an important role of accompaniment and guidance in the sexual education of adolescents. In addition to traditional teaching models, today we are committed to participative methodologies of action. The objective of this work was to promote learning in this subject to students of an institute, comparing different educational methodologies. Three educational workshops (traditional methodology, role-playing and dynamics) were given, and the efficacy of each one was evaluated, using a pretest and posttest questionnaire. The importance of adolescent sexual education in the academic field was confirmed, and some strengths and weaknesses of the traditional and participative methodologies were demonstrated in this process.
\end{abstract}

Keywords: Education for health; Sexuality; Teenagers

\title{
Resumo
}

Profissionais de saúde e educação desempenham um papel importante no apoio e orientar a educação sexual dos adolescentes. Além dos modelos tradicionais de ensino hoje estão comprometidos com metodologias de acção participativos. O objetivo deste trabalho foi incentivar a aprendizagem em que os estudantes da área de uma escola, comparando diferentes metodologias educacionais. Três oficinas educativas (metodologia tradicional, role-playing e dinaminas) foram entregues, e a eficácia de cada um deles, valendo-nos de um questionário pós-teste pré-teste e avaliados. Foi encontrada a importância da educação sexual em adolescentes na academia, e alguns pontos fortes e fracos de metodologias e tradições participativas neste processo foram demonstrados.

Palavras-chave: Educação em saúde; Sexualidade; Adolescentes

\section{Introducción}

La adolescencia es una etapa del ciclo vital de las personas en la que producen múltiples cambios a nivel físico y psicológico, entre ellos, el despertar sexual. De hecho, según la Organización Mundial de la Salud, una de las áreas que requieren especial atención en los adolescentes es la salud sexual, dada la tendencia caza vez más precoz de inicio de relaciones sexuales y problemas que ello puede acarrear. 
Los profesionales sanitarios y de la educación, por cercanía, podemos jugar un importante papel de acompañamiento como de guía durante ese periodo cargado de estímulos (Alpízar, Rodríguez \& Cañete, 2014; Jiménez, Mansilla, Aranda \& Muñoz, 2015; Martins, dos Reis, Oliveira \& Santos, 2013).

La educación para la salud sexual se convierte en una herramienta básica e imprescindible cuyo fin será crear conciencia en esos jóvenes respecto a las consecuencias de la actividad sexual sin protección, así como ayudarlos a explorar valores y sentirse cómodos con su propia sexualidad.

Además de los modelos tradicionales de enseñanza, en los que el profesor forma por medio de métodos magistrales, hoy se apuesta en todo el sistema educativo por metodologías de acción participativas, vinculadas a posicionamientos sociocríticos, en las que los estudiantes sean los responsables de su proceso de aprendizaje (GarcíaCarpintero et al., 2015).

El objetivo de este trabajo fue fomentar el aprendizaje en materia de sexualidad a alumnos de $3^{\circ}$ ESO de un Instituto de León (España), y comparar diferentes metodologías de aprendizaje.

\section{Contextualización}

Los participantes del estudio fueron 155 alumnos, con edades comprendidas entre los 14 y 17 años, alumnos de $3^{\circ}$ de ESO del Instituto de Enseñanza Secundaria "Eras de Renueva” (León, España) (curso académico 2015-2016).

\section{Método}

Al comienzo del estudio, los alumnos cumplimentaron un cuestionario, que había sido elaborado ad-hoc (evaluación pretest), con el fin de determinar las necesidades educativas referentes al tema.

A raíz de la información obtenida se diseñaron tres talleres educativos: el primero consistió en enseñanza con herramientas tradicionales (clase magistral teórica más debate); el segundo, a través de role-playing (dramatización de situaciones de conflicto); y el tercero, por dinámicas de grupo (juegos más debate).

Cada uno de los talleres estuvo compuesto de 4 sesiones, en las que se abordaron los conceptos básicos en materia sexual (anatomía y fisiología), métodos 
anticonceptivos, enfermedades de trasmisión sexual y aspectos psicológicos. Estas tuvieron lugar en el mes de abril de 2016, con una duración de 50 min. cada una, y fueron impartidas por Andrea Aguado Arconada (alumna de $4^{\circ}$ curso del Grado de Enfermería en la Facultad de Ciencias de la Salud de la Universidad de León).

Al finalizar los talleres se pasó nuevamente el cuestionario (evaluación postest).

Para el procesamiento de la información se elaboró una tabla de datos en el programa Microsoft Excel 2007. El estudio estadístico realizado se llevó a cabo con el programa SPSS versión 23. El nivel de significación fue de $\mathrm{p}<0.05$.

En cuanto a las cuestiones éticas, previo al desarrollo de la investigación se solicitó la autorización a los padres de los alumnos para la participación de sus hijos. Se solicitó la aprobación del programa por parte de la Consejería de Educación de Castilla y León.

Se preservó tanto la confidencialidad de los participantes como de los datos obtenidos.

\section{Resultados y discusión}

La muestra estuvo compuesta por 155 alumnos (49,03\% chicas / 50,97\% chicos), con una media de edad de 14,39 años. El 29,68\% de la misma acudió al taller de dinámicas, el 37,42\% al role-playing, y el 32,90\% a enseñanza tradicional.

De entre todos los resultados obtenidos destacamos 2.

En primer lugar, se evidenciaron diferencias significativas entre los grupos pretest y postest, independientemente del tipo de taller llevado a cabo, observándose un aumento de conocimientos además de un menor número de respuestas en blanco. Es decir, que los talleres se convirtieron en este estudio en una herramienta válida y eficaz para educar en temas de sexualidad en los adolescentes.

En segundo lugar, encontramos diferencias estadísticamente significativas respecto a 2 de los 4 ítems evaluados: conceptos básicos en materia sexual, y métodos anticonceptivos. Eso significó que, a la hora de aprender cuestiones sobre anatomía, fisiología y métodos anticonceptivos, los alumnos que acudieron a las sesiones de roleplaying y métodos tradicionales obtuvieron mejores resultados en sus evaluaciones que los que habían acudido a los talleres de dinámicas. 


\section{Conclusiones}

Se ha constatado la importancia que tiene educar en materia de sexualidad a adolescentes en el ámbito académico. Así mismo, se han demostrado algunas de las fortalezas y debilidades de las metodologías tradiciones y de las participativas (roleplaying y dinámicas) en dicho procedo de aprendizaje.

\section{Referencias}

Alpízar, J., Rodríguez, P., \& Cañete, R. (2014). Intervención educativa sobre educación sexual en adolescentes de una escuela secundaria básica. Unión de Reyes, Matanzas, Cuba. Revista Médica Electrónica, 36(5), 572-582.

García-Carpintero, E., Siles, J., Martínez, M.E., Martínez, E., González, S., \& Pulido, R. (2015). El estudiante como protagonista de su aprendizaje: La necesidad del uso del portafolio en enfermería dentro del contexto de educación superior. Index de Enfermería, 24(1-2).

Jiménez, M.I., Mansilla, J., Aranda, E. \& Muñoz, P. (2015). Sexualidad saludable en nuestros jóvenes: un programa de intervención escolar. Pediatría Atención Primaria, 17(65), 33-37.

Martins, D., Dos Reis, M., Oliveira, T., \& Santos, A. (2013). Sexuality in adolescence experience report. Journal of Nursing, 7(1), 820-823. 